\title{
A COMPARISON THEOREM FOR EIGENFUNCTIONS
}

\author{
C. A. SWANSON
}

ABSTRACT. A comparison theorem of Sturm's type is obtained for eigenfunctions of general linear elliptic partial differential operators of second order on bounded domains of $n$-dimensional Euclidean space. The proof is almost immediate from an earlier identity of the author. The theorem is shown to be stronger than some recent theorems of Kurt Kreith.

Let $R$ be a bounded domain in $E^{n}$ whose boundary $\partial R$ has a piecewise continuous unit normal $N$ at each point. The linear elliptic differential equations

$$
\begin{aligned}
& l u=\sum_{i, j=1}^{n} D_{i}\left[a_{i j}(x) D_{j} u\right]+2 \sum_{i=1}^{n} b_{i}(x) D_{i} u+c(x) u=0, \\
& L v=\sum_{i, j=1}^{n} D_{i}\left[A_{i j}(x) D_{j} v\right]+2 \sum_{i=1}^{n} B_{i}(x) D_{i} v+C(x) v=0
\end{aligned}
$$

are under consideration, where $x=\left(x_{1}, \cdots, x_{n}\right) \in R, D_{i}=\partial / \partial x_{i}, i=$ $1, \cdots, n$, the coefficients in (1) and (2) are real and continuous on $\bar{R}$, and the matrices $\left(a_{i j}\right)$ and $\left(A_{i j}\right)$ are symmetric and positive semidefinite in $R$. A comparison theorem will be derived for solutions of (1) and (2) satisfying the following homogeneous boundary conditions on $\partial R$ :

$$
\begin{aligned}
& h[u]=f u+\sum_{i, j=1}^{n} a_{i j} N_{i} D_{j} u=0, \\
& H[v]=F v+\sum_{i, j=1}^{n} A_{i j} N_{i} D_{j} v=0,
\end{aligned}
$$

respectively, where $f$ and $F$ are continuous functions on $\partial R$.

Let $m, M$ be the quadratic functionals defined by

$$
\begin{aligned}
m[u] & =\int_{R}\left[\sum_{i, j} a_{i j} D_{i} u D_{j} u-2 u \sum_{i} b_{i} D_{i} u-c u^{2}\right] d x, \\
M[u] & =\int_{R}\left[\sum_{i, j} A_{i j} D_{i} u D_{j} u-2 u \sum_{i} B_{i} D_{i} u+(g-C) u^{2}\right] d x,
\end{aligned}
$$

Received by the editors September 20,1971 and, in revised form, June 19, 1972. AMS (MOS) subject classifications (1969). Primary 3511.

Key words and phrases. Nonselfadjoint elliptic equation, Sturmian comparison theorem, homogeneous boundary condition, Green formula.

(C) American Mathematical Society 1973 
with $g$ chosen exactly as in [4], [5] and [7]. The variation of $l, h$ is defined to be

$$
V[u]=m[u]-M[u]+\int_{\partial R}(f-F) u^{2} d s .
$$

THEOREM 1. If there exists a nontrivial solution $u$ of (1), (3) such that $V[u]>0$, then every solution of (2), (4) has a zero at some point in $\bar{R}$.

Proof. If $v \neq 0$ in $\bar{R}$, integration of the author's identity [7, p. 326, Equation (9)], or [5, p. 612] over $R$ yields the inequality

$$
M[u]+\int_{\partial R} F u^{2} d s \geqq \int_{R} \sum_{i, j} D_{i}\left(\left(u^{2} / v\right) A_{i j} D_{j} v\right) d x+\int_{\partial R} F u^{2} d s
$$

(which in fact is (10) of [7]). However, the right side of (5) is equal to $\int_{\partial R}\left(u^{2} / v\right) H[v] d s$ by Green's formula, which is zero by (4). Also Green's formula gives

$$
m[u]+\int_{\partial R} f u^{2} d s=-\int_{R} u l u d x+\int_{\partial R} u h[u] d s=0
$$

and subtraction of (5) from (6) yields the contradiction $V[u] \leqq 0$.

The preceding simple proof shows that Kreith's statement [4] "the technique of [5] seems to be limited to the case where $u(x)=0$ on $\partial R$ " is unfounded.

When $\partial R \in C^{2}$, it is known from the Hopf maximum principle that $v$ and $\partial v / \partial N$ cannot both be zero at any point of $\partial R$, and hence the conclusion of Theorem 1 can be strengthened to " $v$ has a zero in $R$." In the case of the boundary condition $u=0$, Allegretto [1] and the author [8] have derived the strong conclusion for domains with $C^{1}$ boundaries, for example.

Kreith's theorem $[4$, p. 549] replaces the hypothesis $V[u]>0$ of Theorem 1 by $W[u]>0$, where

$$
W[u]=V[u]+\int_{R}\left(2 u \nabla u \cdot \boldsymbol{p}+u^{2} \boldsymbol{\nabla} \cdot \boldsymbol{p}\right) d x-\int_{\partial R} \boldsymbol{p} \cdot N u^{2} d s
$$

for some $C^{1}$ vector function $p$ on $\bar{R}$. Actually, $W[u]=V[u]$ for an arbitrary $C^{1}$ vector function $p$ by Green's formula, so that Kreith's hypothesis about the existence of $\boldsymbol{p}$ is redundant. Various corollaries of Theorem 1 (which are weaker than Theorem 1) given by Kreith follow by specializing $\boldsymbol{p}$ so that a certain quadratic form is positive semidefinite in $R$ [4, p. 548].

In the special case that (3) is replaced by the boundary condition $u(x)=0$ on $\partial R$, Theorem 1 reduces to the author's earlier comparison theorem [5], also stated as Corollary 1 of [4]. Another comparison theorem of Kreith ([3] and [4, Corollary 2]) is the special case that the derivative terms in $V[u]$ are replaced by a lower bound $-g_{2}(x) u^{2}(x)$ in $R$. 
If $\boldsymbol{b}$ and $\boldsymbol{B}$ happen to be differentiable functions, integration by parts transforms $V[u]$ to the form

$$
V[u]=\int_{R}\left[\sum_{i, j}\left(a_{i j}-A_{i j}\right) D_{i} u D_{j} u+(C-c-g+\nabla \cdot(b-B)) u^{2}\right] d x
$$

yielding various immediate corollaries of Theorem 1 ([4], [5], [7]).

Theorem 1 can easily be extended to unbounded domains $R$ by the technique of [7], to quasilinear differential equations as in [8], and to differential inequalities as in [7]. We remark that Theorem 1 in the selfadjoint case was obtained in 1966 by the author [6] along with an extension to elliptic operators of even order.

A generalization of some recent theorems of Dunninger and Weinacht [2] also follows immediately from Theorem 1, i.e. from the author's identity $([5$, p. 612$]$ and $[7$, p. 327]) in the special case that every entry of the matrices $\left(a_{i j}\right)$ and $\left(A_{i j}\right)$ vanishes on a subdomain $S^{\prime}$ of $\partial R$. Then obviously (5) and (6) still hold if the boundary conditions (3) and (4) hold only on $\partial R-S^{\prime}$ (with $f=F=0$ on $S^{\prime}$ ).

COROLlaRY. Let $S^{\prime} \subset \partial R$ be the "singular part" of $\partial R$, i.e. $a_{i j}=A_{i j}=0$ on $S^{\prime}$ for $i, j=1,2, \cdots, n$. Then Theorem 1 remains true if the boundary conditions (3) and (4) hold only on $\partial R-S^{\prime}$.

As in [7], the same conclusion holds if (1), (2), (3), and (4) are replaced by the inequalities $u l u \geqq 0, v L v \leqq 0, u h[u] \leqq 0$, and $v H[v] \geqq 0$, respectively, since (5) remains true and (6) is replaced by $m[u]+\int_{\partial R} f u^{2} d s \leqq 0$. The main Theorem 3.1 of Dunninger and Weinacht [2] is the following special case of the corollary:

$$
\begin{aligned}
b_{i} & =B_{i}=0, \quad i=1, \cdots, n ; \quad g=0, \\
a_{i j} & =y^{k} \tilde{a}_{i j}, \quad A_{i j}=y^{k} \tilde{A}_{i j} \quad \text { if } i, j=1, \cdots, n-1=m ; \\
a_{i n} & =A_{i n}=0 \quad \text { if } i=1, \cdots, n-1 ; \\
a_{n n} & =A_{n n}=y^{k} ; \quad c=y^{k} \tilde{c}, \quad C=y^{k} \tilde{C} ; \\
f & =y^{k} \tilde{f}, \quad F=y^{k} \tilde{F} ; \quad \text { where } y=x_{n} .
\end{aligned}
$$

In this case, the variation $V[u]$ reduces to

$$
\begin{aligned}
V[u]= & \int_{R} y^{k}\left[\sum_{i, j=1}^{n-1}\left(\tilde{a}_{i j}-\tilde{A}_{i j}\right) D_{i} u D_{j} u+(\tilde{C}-\tilde{c}) u^{2}\right] d x \\
& +\int_{S_{2}} y^{k}(\tilde{f}-\tilde{F}) u^{2} d s,
\end{aligned}
$$

where $S_{2}$ is the component of $\partial R$ on which $f$ and $F$ in the boundary conditions (3) and (4) are finite and nonzero. (The boundary condition $u=0$ 
corresponds to $f=\infty$ by the usual convention.) Other theorems of Dunninger and Weinacht are special cases or easy consequences of the above results. (On [2, p. 55], the author's method ([5], [7]) of using a quadratic form in $n+1$ variables is mentioned without reference.)

\section{REFERENCES}

1. W. Allegretto, A comparison theorem for nonlinear operators, Ann. Scuola Norm. Sup. Pisa (3) 25 (1971), 41-46.

2. D. R. Dunninger and R. J. Weinacht, Separation and comparison theorems for classes of singular elliptic inequalities and degenerate elliptic inequalities, Applicable Anal. 1 (1971), 43-55.

3. $\mathrm{K}$. Kreith, A comparison theorem for general elliptic equations with mixed boundary conditions, J. Differential Equations 8 (1970), 537-541. MR 42 \#646.

4. - A class of comparison theorems for nonselfadjoint elliptic equations, Proc. Amer. Math. Soc. 29 (1971), 547-552.

5. C. A. Swanson, A comparison theorem for elliptic differential equations, Proc. Amer. Math. Soc. 17 (1966), 611-616. MR 34 \#1663.

6. - A generalization of Sturm's comparison theorem, J. Math. Anal. Appl. 15 (1966), 512-519. MR 34 \#4656.

7. - An identity for elliptic equations with applications, Trans. Amer. Math. Soc. 134 (1968), 325-333. MR 38 \#400.

8. - Comparison theorems for elliptic differential systems, Pacific J. Math. 33 (1970), 445-450. MR 41 \#7255.

Department of Mathematics, Uniersity of British Columbia, Vancouver, British Columbia, Canada 